\title{
TERZA MISSIONE E SERVICE LEARNING NEL MODELLO DELLE CATTEDRE SCHOLAS
}

Carina Rossa ${ }^{58}$

\section{Sintesi}

Tutti riconoscono le funzioni della didattica e della ricerca come missioni identitarie dell'università. Nel tempo si è introdotta una terza missione che riguarda il rapporto con il territorio. Il nuovo millennio porta un nuovo paradigma sulla terza missione in America Latina, si tratta dell'approccio di Responsabilità Sociale Universitaria (RSU). Questo orientamento si definisce innanzitutto per la gestione degli impatti dell'organizzazione nel proprio intorno umano, sociale, economico, naturale, in funzione dello sviluppo sostenibile. Alcune proposte pedagogiche, come il Service Learning, si propongono come approcci adeguati a questo modello di università nel momento in cui gli attori esterni lavorano insieme agli agenti interni all'università per attivare progetti di sviluppo, produrre nuove conoscenze socialmente utili e formare futuri professionisti attorno a valori che aiutano a migliorare la qualità di vita della popolazione, promuovendo la inter e trans-disciplinarietà. La sfida delle Cattedre Scholas è quella di supportare una rete mondiale di università impegnate socialmente, una rete come reale strumento di trasformazione dell'umanità.

Parole chiave: Terza missione. Responsabilità Sociale Universitaria. Service Learning. Cattedre Scholas.

\section{TERCEIRA MISSÃO E APRENDIZAGEM SOLIDÁRIA NO MODELO DAS CÁTEDRAS SCHOLAS}

\section{Resumo}

Todos reconhecem as funções de ensino e pesquisa como missões de identidade universitária. Com o tempo, uma terceira missão foi introduzida, que diz respeito ao relacionamento com o território. O novo milênio traz um novo paradigma sobre a terceira missão na América Latina, é a abordagem da Responsabilidade Social Universitária (RSU). Essa orientação é definida principalmente para a gestão dos impactos da organização em seu entorno humano, social, econômico e natural, em função do desenvolvimento sustentável. Algumas propostas pedagógicas, como a Aprendizagem Solidária, se propõem como abordagens apropriadas para esse modelo de universidade quando os atores externos trabalham em conjunto com os agentes internos da universidade para ativar projetos de desenvolvimento, produzir novos conhecimentos socialmente úteis e treinar futuros profissionais a respeito de valores que ajudam a melhorar a qualidade de vida da população, promovendo a inter e a transdisciplinaridade. O desafio das Cátedras Scholas é a de suportar uma rede mundial de universidades

\footnotetext{
${ }^{58} \mathrm{PhD}$ in Psicologia dello Sviluppo e Trasformazione sociale, membro del Consiglio Direttivo della Fondazione Pontificia Scholas Occurrentes, ricercatrice Istituto Universitario Sophia, Firenze.
} 
socialmente engajadas, uma rede como um instrumento real para transformar a humanidade.

Palavras-chave: Terceira missão. Responsabilidade Social Universitária. Aprendizagem Solidária. Cátedras Scholas.

\title{
THIRD MISSION AND SERVICE LEARNING IN THE MODEL OF SCHOLAS CHAIRS
}

\begin{abstract}
Everyone recognizes the functions of didactics and research as university identity missions. Over time, a third mission has been introduced which concerns the relationship with the territory. The new millennium brings a new paradigm on the third mission in Latin America, it is the approach of University Social Responsibility (RSU). This orientation is defined above all for the management of the impacts of the organization in its human, social, economic, natural surroundings, according to sustainable development. Some pedagogical proposals, such as Service Learning, are proposed as appropriate approaches to this model of university when the external actors work together with the agents inside the university to activate development projects, produce new socially useful knowledge and train future professionals around values that help improve the quality of life of the population, promoting inter and trans-disciplinarity. The challenge of Scholas Chairs is to support a worldwide network of socially engaged universities, a network as a real instrument for transforming humanity.
\end{abstract}

Keywords: Third mission. University Social Responsibility. Service Learning. Scholas Chairs.

\section{Introduzione}

La UNEB, Campus III,_in Juazeiro-BA, ha realizzato nel mese di Agosto 2019 il I Colóquio Brasil Itália con il tema 'Educazione contestualizzata e dialogo interculturale nella implementazione di nuove strategie di internazionalizzazione e di responsabilità sociale universitaria' nel quale si è presentata la proposta pedagogica del Service Learning come sviluppo della terza missione e dell'internazionalizzazione. In particolare, si è esposto il modello di rete delle Cattedre Scholas come ambito nel quale convivono questi elementi distintivi.

Le Cattedre Scholas rappresentano uno spazio accademico qualificato che si propone di essere punto di riflessione e di azione, collegato in una rete in cui ogni soggetto (docenti, ricercatori, studenti) si arricchisce nell'incontro con l'altro attraverso i vincoli che si sviluppano tra le diverse università, le scuole e i progetti presenti nella piattaforma 
Scholas.social. In questo momento sono 103 università di 35 paesi dei 5 continenti, rappresentando università pubbliche e private e di diverse religioni.

Il Service Learning è una proposta pedagogica che cerca di soddisfare i bisogni veri e sentiti di una comunità, dove i giovani sono i protagonisti in tutte le sue fasi, dalla pianificazione alla valutazione, articolata in modo intenzionale con i contenuti di apprendimento. Una proposta che parte dalla convinzione che è nella comunità e insieme alla comunità che si generano i saperi e non nelle discussioni accademiche all'interno dell'aula e svincolate dalla realtà. Questa stessa offre un contributo alla riflessione promuovendo il ritorno dell'apprendimento, teorizzando su ciò che si fa e quello che si produce.

Il Service Learning consente la messa in pratica del valore della solidarietà, dell'impegno nel sociale, l'approccio verso la povertà e le sue cause profonde, l'impegno con l'altro ed il rapporto fraterno che porta a condizioni di vita più giuste. Consente il protagonismo dei giovani che, con tutto il potenziale della loro giovinezza ed i propri ideali, arricchiti con le conoscenze accademiche, saranno i costruttori dello sviluppo locale in un mondo globalizzato.

\section{La Terza Missione: uno sguardo storico}

Al di là della lunga tradizione esistente, soprattutto in America Latina, della extension universitaria e dell'esortazione fatta dalla UNESCO nel lontano 1998 (UNESCO, 1998), la dimensione di responsabilità ed impegno sociale universitario in senso integrale non è ancora approdata completamente nelle istituzioni di educazione superiore.

Tutti riconoscono le funzioni della didattica e della ricerca come missioni identitarie dell'università. Nel tempo si è introdotta una terza missione, qui chiamata extension, che riguarda il rapporto con il territorio. Per capire meglio come si è sviluppata la consapevolezza dell'inserimento di questo terzo pilastro occorre riportare un piccolo cenno storico.

L'università alle sue origini, nel Medioevo, è costituita al servizio della produzione e trasmissione del sapere secondo il 'metodo scolastico' attraverso il quale lo studente viene avviato a percorrere un cammino intellettuale che segue queste tappe: la lectio (lettura), la quaestio (individuazione di problemi), la disputatio (disputa interpretativa) e la determinatio (sintesi finale). In questo modello di università il pilastro 
principale è pertanto la didattica. Alla fine del secolo XVIII ed inizi del secolo XIX, l'università tedesca stabilisce invece come pilastro fondamentale la ricerca. Sarà l'approccio statunitense nel secolo XX a collocare come terzo pilastro il servizio alla comunità.

Nelle origini possono distinguersi due modelli universitari: il modello di Bologna (universitas scholarium) ed il modello di Parigi (universitas magistrorum). La pressione che esercitano gli studenti cercando professori, fa sorgere l'Università di Bologna che si caratterizzerà per una grande partecipazione degli stessi studenti nella governance ed amministrazione dell'Ateneo. Contrariamente l'Università di Parigi avrà come obiettivo soddisfare i bisogni dottrinali della Chiesa Cattolica. Una delle conseguenze, dunque, della Rivoluzione Francese sarà la chiusura di tutte le università in Francia considerate rifugio di privilegiati. L'insegnamento superiore, di conseguenza, viene riorganizzato secondo le scuole specialistiche, come la Scuola Politecnico di Parigi. L'educazione passa in mano allo Stato e si stabilisce un modello utilitario dove prevale l'insegnamento professionale. 'L'organismo statale stabilisce la morale pubblica da 'inculcare' trasformandola in una istituzione centralizzata, burocratica e gerarchica." (OYARZN, 1976, p.7). Incomincia così la frammentazione del sapere in discipline separate, allontanandosi dalla concezione unitaria del sapere tipica delle università del medioevo.

Questo modello francese, istituito da Napoleone, è seguito in America Latina con lo scopo di preparare professionisti per l'amministrazione pubblica e per la società. L'attività di ricerca, invece, era riservata esclusivamente per l'accademia.

Nei secoli XIX e XX le università si concentrano sullo studio delle scienze, escludendo le classi più popolari. Secondo Morales (2010) attorno al 1810 con la creazione dell'Università di Berlino, nasce la concezione dell'università moderna. Questa istituzione mette come fondamento dell'insegnamento non soltanto le conoscenze attendibili, ma piuttosto cerca di dimostrare come si arriva a tali saperi, si mette in evidenzia, cioè, il processo scientifico della mente. L'università passa, quindi, da essere un centro per l'insegnamento a convertirsi in un centro per la ricerca. Appare il modello 'humboldtiano' ovvero un modello di università che coniuga ricerca e didattica nel nome del progresso della nazione, con l'esplicito mandato di formare l'elite. All'inizio del secolo XX il modello tedesco aveva gettato le basi nelle università più importanti del mondo. Le università tedesche erano molto prestigiose ed i suoi professori riconosciuti a livello internazionale. 
Attorno al secolo XIX si affacciano i primi vestigi della terza missione: la extension o 'collegamento con il territorio' con lo scopo di avvicinare chi, per la sua condizione economica, era escluso del sistema universitario. Nel 1790, in Inghilterra, di fronte alla rivoluzione industriale, incominciano a svilupparsi programmi di formazione per adulti. Il primo College di classe operaria viene fondato nel 1842 in Sheffield, Inghilterra. Nel 1867, nell'Università di Cambridge, nasce il primo programma di extension (LABRANDERO, I.M.Y.; SANTANDER, L. C., 1983) con l'intenzione di coprire il bisogno di formazione della popolazione dei paesi accanto, offrendo un ciclo di conferenze. Lo scopo era avvicinare il sapere al popolo, abbandonando il precetto che indicava che per accedere all'università si doveva essere ricco o nobile. Germania, Ungheria, Russia e Spagna seguono queste iniziative in alcune delle loro università.

Tuttavia, la più importante azione considerata come prodromo della extension si realizza negli Stati Uniti, nel 1862, nel Land Grant Colleges, estendendo l'educazione superiore alla maggior parte della popolazione con un modello agricolo ed industriale. Le scuole più prestigiose degli Stati Uniti seguono questo modello provocando una rottura con l'approccio d'elite ed intellettualistico fino ad allora prevalente. Queste scuole mettono in rilievo il nuovo paradigma di rapporto con il territorio.

Contemporaneamente le università in America Latina, create secondo il modello della Universidad de Salamanca, continuano con uno stile d'insegnamento d'elite. La vita culturale universitaria ed il rapporto con il territorio è ridotto a celebrazioni occasionali di alcuni atti culturali rivolti ad un pubblico scelto proveniente dallo stesso strato sociale di appartenenza dei professori e studenti. Nel 1908 a Montevideo (Uruguay) si realizza il 'Primo incontro internazionale di studenti americani'. Nel manifesto viene espressa l'esigenza di stabilire programmi di extension universitaria e sarà nel 1918, a Cordoba (Argentina), che le università latinoamericane, nel cosiddetto 'movimento o riforma di Cordoba' propizieranno l'accesso agli studi superiori delle classi medie facendo nascere la 'missione sociale dell'Università' come una terza missione accanto all'insegnamento ed alla ricerca. Questa riforma enfatizzerà il diritto dell'educazione per tutti, il dialogo tra università e società e l'obbligo di condividere la cultura ed il sapere con chi, per motivi sociali, non può accostarsi allo studio. 


\section{Dalla extension alla responsabilità sociale universitaria}

Lo sviluppo della extension, sin dalla nascita, è stato legato sia al grado e tipo di rapporto dell'università con la società, sia ai principi filosofici che nutrivano l'educazione superiore. Le prime interazioni nascono con il sorgere della rivoluzione industriale, tuttavia, questa apertura verso la società non sembra rispondere agli interessi di tutta la popolazione, bensì a chi sostenta il potere, visto che le attività incominciano come risposta alla crescita industriale dei potenti dell'epoca. In America, invece, le idee di progresso dei movimenti indipendentisti influenzano in modo diverso. L'enfasi viene posta sulla aspirazione degli emarginati della società ad accedere all'insegnamento superiore. Nel 1918, con la riforma di Cordoba (Argentina), si provoca una rottura con i vecchi canoni universitari ed appare una terza missione universitaria: la missione di mettersi al servizio della società ed occuparsi dei problemi del territorio.

Nella decade degli anni '50 si sviluppa una concettualizzazione della extension che garantisce la sistematizzazione e coerenza della sua funzione sociale. Ciò nonostante, viene considerata come una azione unilaterale nella quale l'università, custodia della cultura, offre o trasmette al popolo i suoi saperi, dall'alto in basso. Nel 1972 viene fatta una nuova riflessione sulla missione dell'università, soprattutto sull'atteggiamento che le istituzioni educative hanno verso il territorio ed incomincia a profilarsi uno stile di extension diverso, incominciando un processo di interazione tra università e comunità. Nasce dunque un modello di reciprocità attraverso il quale si scopre quanto la società può contribuire alla trasformazione e al perfezionamento dell'università.

Il nuovo millennio porta un altro paradigma sulla terza missione in America Latina, si tratta dell'approccio di Responsabilità Sociale Universitaria (RSU). Questo orientamento si definisce innanzitutto per la gestione degli impatti dell'organizzazione nel proprio intorno umano, sociale, economico e naturale, in funzione dello sviluppo sostenibile.

Secondo la RSU sono quattro i principali impatti che genera una istituzione di educazione superiore: 1) impatti che provengono dalla stessa organizzazione, dal proprio campus e personale (attività lavorativa e dell'ambiente); 2) impatti che derivano dalla formazione (stili della didattica); 3) impatti che derivano dalla produzione dei saperi, presupposti epistemologici, decisioni accademiche (cognitivi e di ricerca); 4) impatti che provengono dal rapporto con il territorio, le reti, la partecipazione sociale, economica e 
politica dell'università (extension) (VALLAEYS, F.). Questi sono gli effetti che l'università deve imparare a gestire in forma socialmente responsabile.

La RSU si può definire allora come "Una politica di qualità etica della performance della comunità educativa (studenti, docenti e personale amministrativo) attraverso la gestione responsabile degli impatti educativi, cognitivi, del lavoro e dell'ambiente che l'università genera, in un dialogo partecipativo con la società per migliorare l'aspetto accademico e promuovere lo sviluppo umano integrale" (Op.cit IBIDEM).

In questa prospettiva di terza missione vista dal paradigma della RSU è benvenuta la Enciclica Laudato Si di Papa Francesco (SANTO PADRE, F., 2015) che propone una conversione ecologica integrale, un cambiamento negli stili di vita che si rende necessario anche nelle istituzioni di educazione superiore. Essa porta ad una trasformazione della gestione dell'università a tutto campo, in modo responsabile ed etico, invitando a modificare le pratiche interne ed esterne, gli stili di produzione e di trasmissione del sapere in dialogo con la realtà che appare di per sé interdisciplinare.

Alcune proposte pedagogiche, come il Service Learning, si propongono come approcci adeguati a questo modello di università nel momento in cui gli attori esterni lavorano insieme agli agenti interni all'università per attivare progetti di sviluppo, produrre nuove conoscenze socialmente utili e formare futuri professionisti attorno a valori che aiutano a migliorare la qualità di vita della popolazione, promuovendo la inter e trans-disciplinarietà.

\section{Il Service Learning}

Le attività di Service Learning sono presenti nell'ambito universitario sin dalla sua nascita negli anni Sessanta negli Stati Uniti e fanno parte integrale dei programmi di extension e di RSU ormai a livello mondiale.

Esistono numerose definizioni di Service Learning. Tra le voci più autorevoli in questo campo troviamo Andrew Furco, noto ricercatore che afferma: "Il Service-learning cerca di coinvolgere gli studenti in un'attività che combina il servizio alla comunità e l'apprendimento accademico. Siccome i programmi di Service-learning si inseriscono nei corsi ufficiali (a scuola o all'università), le attività di servizio si impostano sempre su specifici contenuti curriculari” (FURCO, A.; SHELLEY H. B., p.5). 
Gli spagnoli Puig e Rovira si sono preoccupati di sottolineare la novità e la specificità del Service Learning, affermando:

L'apprendimento-servizio $(\mathrm{ApS})$ è un'attività complessa che integra il servizio alla comunità con l'apprendimento. È una proposta innovatrice, anche se si basa su elementi molto conosciuti e di lunga tradizione come il servizio volontario alla comunità e l'apprendimento di conoscenze, abilità e valori scolastici. L'innovazione, o novità, non è riscontrabile nelle parti che compongono l'apprendimento e il servizio, ma nello stretto collegamento tra il servizio e l'apprendimento in un'unica attività articolata, coordinata e coerente. (PUIG ROVIRA, J. M.; PALOS RODRÍGUEZ, J., 2006, p.61).

La definizione elaborata da Maria Nieves Tapia, con il Centro Latino-americano de Aprendizaje y Servicio Solidario CLAYSS, evidenzia le tre caratteristiche programmatiche del Service Learning, vale a dire il 'servizio solidale', il 'protagonismo degli studenti' e la 'articolazione curriculare'. Questa, forse, è una tra le poche definizioni che mette in rilievo l'aspetto della partecipazione protagonistica dei bambini, dei ragazzi e dei giovani come aspetto intrinseco di essa:

Servizio solidale destinato a soddisfare i bisogni veri e sentiti della comunità, con il protagonismo attivo degli studenti in tutte le tappe del progetto (dalla pianificazione sino alla valutazione) e articolato intenzionalmente con i contenuti di apprendimento (contenuti curriculari o formativi, riflessione, sviluppo delle competenze per la cittadinanza attiva, il lavoro e la ricerca) (TAPIA, M., 2015, p.32) ${ }^{59}$

Numerosi autori confermano che il Service Learning è una pedagogia o una filosofia dell'educazione.

[...] È un modo di pensare l'educazione e l'insegnamento (una filosofia) con i corrispondenti strumenti e strategie d'insegnamento, una pedagogia, quindi, che sollecita gli studenti ad imparare e a crescere attraverso l'attiva partecipazione nelle attività di servizio per raggiungere gli obiettivi definiti dalle organizzazioni[...]Il Service Learning non è solo un programma, ma anche una filosofia; cioè, un modo di capire la crescita umana, un modo di spiegare la creazione di vincoli sociali e un cammino che dà vita a comunità umane più giuste $\mathrm{e}$ con una migliore convivenza" (PUIG ROVIRA, J. M. et al, 2011, pag.10).

“(È) una filosofia di crescita umana e di senso, una visione sociale, un modo di avvicinarsi alla comunità e un modo di conoscere" (KENDALL, 1990, p.23 IN TAPIA, 2015, p.33).

${ }^{59}$ Traduzione di Carina Rossa. 
Parliamo di Service Learning, quindi, quando c'è l'intersezione di due intenzionalità, in altre parole, quando nella realizzazione di un progetto sono contemporaneamente presenti l'intenzionalità pedagogica e l'intenzionalità di servizio.

Sigmon intende il Service Learning come un apprendimento che può essere il frutto dell'attività di servizio e che risulta utile sia per chi fornisce il servizio sia per chi lo 'riceve', in quanto stiamo parlando di soggetti attivi che riescono ad 'imparare' da questa esperienza (FURCO, A., 1996, pp.2-6). Secondo Sigmon il Service Learning avviene soltanto quando tutte le parti coinvolte apprendono qualcosa; ciò si manifesta con i risultati di servizio. È quest'ultimo concetto che fornisce la chiave per stabilire l'equilibrio tra gli obiettivi di servizio e quelli di apprendimento e per distinguere il Service Learning da altri tipi di programmi di servizio o di educazione esperienziale (volontariato, tirocinio, lavoro di campo, ecc.).

Un buon progetto di apprendimento-servizio permette, in prima istanza, di migliorare la qualità dell'apprendimento, ma contribuisce anche al miglioramento della qualità di vita di una comunità. Consente ai giovani di imparare contenuti di apprendimento curricolare mentre svolgono dei compiti importanti e responsabili nella comunità e nella istituzione educativa stessa.

In questo modo si stabilisce un 'circolo virtuoso' nel quale l'applicazione dei saperi migliora la qualità del servizio offerto alla comunità e l'azione di servizio, inoltre, coincide con una migliore formazione integrale, spronando una nuova produzione di conoscenze.

$\mathrm{Al}$ di là della complessità e della varietà del contesto globale, dell'enfasi e delle variazioni poste da ogni autore, negli ultimi anni la comunità accademica concorda nel riconoscimento dei tre tratti fondamentali o 'programmatici' che contraddistinguono il Service Learning:

- Lo sviluppo di attività di servizio solidale volte ad offrire risposte efficaci per la soluzione dei problemi concreti della comunità.

- Il protagonismo delle studentesse e degli studenti nella pianificazione, nello sviluppo e nella valutazione del progetto: si tratta di una proposta di apprendimento attivo ed è per questo che gli studenti, più che i professori, devono essere i protagonisti e fare proprie le attività.

- L'integrazione intenzionale delle pratiche solidali con i contenuti di apprendimento e di ricerca inclusi nel curriculum. Questo è l'ambito di protagonismo dei docenti. 


\section{Il modello della Cattedre Scholas}

Nel 2016 nasce il programma Cattedre Scholas, promosso dalla Fondazione Pontificia Scholas Occurrentes e rivolto alle università che aderiscono, studiano e promuovono la pedagogia di Papa Francesco. Le Cattedre rappresentano degli spazi accademici qualificati che si propongono di essere dei punti di riflessione e di azione, collegati in una rete, in cui ogni soggetto (docenti, ricercatori, studenti) si arricchisce nell'incontro con l'altro, attraverso i vincoli che si sviluppano tra le diverse università, le scuole e i progetti presenti nella piattaforma Scholas.social.

Attualmente sono 103 le università di 35 paesi dei 5 continenti, che partecipano rappresentando università pubbliche e private e di diverse religioni.

Il pensiero di Papa Francesco, espresso in tanti incontri, discorsi, documenti e pubblicazioni, è particolarmente ricco di profondità pedagogica e di attenzione alla comunità sociale, così che lo stesso Papa Francesco può essere considerato un grande Maestro dei nostri giorni, la cui autorevolezza è riconosciuta ben oltre i confini della chiesa cattolica e, perfino, da quanti si proclamano cristiani, per abbracciare persone di ogni credo e idealità. Il suo linguaggio è semplice e immediato, accessibile a tutti. Il suo messaggio parla alle donne e agli uomini di oggi e offre, in un mondo dilaniato dalle guerre, dalle ingiustizie, dalle incomprensioni e dalle intolleranze, la prospettiva per l'edificazione di quel nuovo umanesimo di cui tutti abbiamo bisogno. La ricchezza di tale pensiero merita di essere non solo conosciuta e fatta conoscere, ma attentamente studiata e concretamente 'ascoltata'. Se l'impegno di un ascolto attento e di un approfondimento intelligente interpella non solo i singoli, ma anche le istituzioni, questo compito non può essere disertato da quell'istituzione di studio, ricerca e formazione per eccellenza che è l'università (FIORIN, I.; CULCASI, I., 2018).

Nella loro missione le Cattedre prendono spunto della costituzione Veritatis Gaudium (COSTITUZIONE APOSTOLICA, 2018) ispirandosi a questi principi o criteri:

1. La 'mistica del noi': che si fa lievito di quella fraternità universale «che sa guardare alla grandezza del prossimo, che sa sopportare le molestie del vivere insieme. Di qui l'imperativo ad ascoltare nel cuore e a far risuonare nella mente il grido dei poveri e della terra, per dare concretezza alla dimensione sociale, l'opzione per gli ultimi, per quelli che la società scarta e getta via, propiziando 'una spiritualità della solidarietà globale'.» (IBIDEM, p.6) 
2. Il dialogo a tutto campo: non come mero atteggiamento tattico, ma come esigenza intrinseca per fare esperienza comunitaria della gioia della verità e per approfondirne il significato e le implicazioni pratiche in generosa e aperta sinergia con tutte le istanze positive che fermentano la crescita della coscienza umana universale, è un'autentica cultura dell'incontro, una cultura anzi, possiamo ben dire, dell'incontro tra tutte le autentiche e vitali culture, grazie al reciproco scambio dei propri rispettivi doni. In questo senso favoriamo il dialogo ecumenico e interreligioso e con coloro che aderiscono ad altre convinzioni religiose o umanistiche.

3. L'inter e la trans-disciplinarietà: principio vitale e intellettuale dell'unità del sapere nella distinzione e nel rispetto delle sue molteplici, correlate e convergenti espressioni.

4. La necessità urgente di 'fare rete': tra le diverse istituzioni, in ogni parte del mondo, attivando con decisione le opportune sinergie anche con le istituzioni accademiche dei diversi paesi e con quelle che si ispirano alle diverse tradizioni culturali e religiose, dando vita al contempo a centri specializzati di ricerca finalizzati a studiare i problemi di portata epocale che investono oggi l'umanità, giungendo a proporre opportune e realistiche piste di risoluzione.

Ogni professore nel suo insegnamento, ogni gruppo di professori all'interno della propria università, possono far nascere una Cattedra Scholas e iniziare a sviluppare un progetto di ricerca accademica e di impegno educativo, incontrando realtà esterne all'università che manifestano una domanda di aiuto.

La novità accademica introdotta dalle Cattedre non consiste tanto nel fatto che l'università si apra alla realtà esterna, quanto nel tipo di rapporto che si intende instaurare. Solitamente, però, la relazione che si instaura con la realtà è di tipo funzionale e risponde alle esigenze interne all'università stessa, che ha bisogno di procurarsi risorse dall'ambiente esterno. Le Cattedre invertono questa logica, l'università non esce dalle proprie mura per procurarsi risorse, ma per essere risorsa per la comunità. Al paradigma utilitaristico contrappongono il paradigma del servizio (FIORIN, I.; CULCASI, I., 2018).

Nelle Cattedre il dialogo tra le diverse religioni assume un rilievo particolare. La composizione multiculturale e multireligiosa che si trova nelle tante università che aderiscono, rappresenta una grande risorsa come fonte di arricchimento e di costruzione di un mondo più solidale e pacifico. 


\section{Conclusione}

A proposito dell'educazione, Papa Francesco usa spesso un'espressione molto forte: "Non si cambia il mondo se non si cambia l'educazione". Siamo consapevoli del valore enorme che ha l'educazione per cambiare il mondo ma allo stesso tempo sappiamo quanto ancora essa deva cambiare per essere in grado di trasformare la società. Le università hanno fatto un percorso storico importante maturando il profilo di una istituzione sempre più permeabile al territorio in una logica di servizio.

Il paradigma della Responsabilità Sociale Universitaria può e deve in questo momento offrire una risposta perché questo cambiamento non sia un maquillage istituzionale di buone intenzioni, bensì porti ad uno stile di vita etico e responsabile tra tutti gli attori della comunità educativa modificando i rapporti, la gestione interna, la didattica, la produzione del sapere ed il rapporto con il territorio. La proposta pedagogica del Service Lerning costituisce una possibilità sistematica, ordinata e collaudata che può attivare i meccanismi interni ed esterni che consentono di mettere in moto questa politica di responsabilità sociale.

In questo contesto la sfida delle Cattedre Scholas sarà quella di supportare una rete mondiale di università impegnate socialmente, una rete come reale strumento di trasformazione dell'umanità.

\section{Bibliografia}

COSTITUZIONE APOSTOLICA «Veritatis gaudium» di Papa Francesco circa le Università e le Facoltà ecclesiastiche, Bollettino Sala Stampa della Santa Sede 29.01.2018. 2018. Vaticano: Disponível no site: https://press.vatican.va/content/salastampa/it/bollettino/pubblico/2018/01/29/0083.html

FIORIN, I.; CULCASI, I. Le Cattedre Scholas. In: Revista Educatio Catholica Anno IV 1/2018. Città del Vaticano: Libreria Vaticana, 2018.

FURCO, A.; SHELLEY H. B., eds. Service-Learning: The Essence of the Pedagogy. Greenwich, CT: Information Age Publishing Inc., 2002.

- Service-learning: A balanced approach to experiential education, in Expanding boundaries: service and learning, edited by B. Taylor, Washington, DC: Corporation for National Service, pp. 2-6, 1996.

LABRANDERO I.M.Y.; SANTANDER, L. C. Extensión académica: una función del sistema universitario. Mexico: ANUIES-Revista de la Educación Superior, N 47, 1983. 
MORALES, M.; MIRA, G.; ARIAS, M. Enfoques y retos de la función de extensión universitaria como mecanismo de integración: Universidad, Empresa, Estado. II Congreso Internacional de Gestión Tecnológica e Innovación. Bogotá: D. C., Colombia, 2010.

OYARZUN, Galo Gómez. La Universidad: sus orígenes y evolución. Deslinde, UNAM, 1976.

PUIG ROVIRA, J. M.; PALOS RODRÍGUEZ, J. Rasgos pedagógicos del aprendizajeservicio, Cuadernos de Pedagogía, núm. 357, magg, 2006.

et al. Aprendizaje-servicio y educación para la ciudadanía. In: Revista de Educación, número extraordinario, pp. 45-67, 2011.

SANTO PADRE, Francesco. Laudato si'. Lettera Enciclica sulla cura della casa comune. Città del Vaticano: LEV, 2015.

TAPIA, M. El compromiso social como pedagogía. Aprendizaje y solidaridad en la escuela. Bogotá: Ed. CELAM, 2015.

UNESCO. Conferencia Mundial sobre la Educación Superior. La Educación Superior en el siglo XXI. Paris: Visión y Acción, 9 de octubre de 1998.

VALLAEYS, F. ¿Qué es la Responsabilidad Social Universitaria? Disponível em http://creasfile.uahurtado.cl/RSU.pdf 